\title{
The Relation between the Masses and Luminosities of the Stars.
}

\author{
By Prof. A. S. Eddington, F.R.S.
}

A THEORY of the physical conditions in the A interior of a star should, if sufficiently complete, lead to formulæ determining the total radiation emitted, and hence the absolute brightness. In the simplest case-that of a star in the condition of a perfect gas-considerable progress appears to have been made towards the solution of this problem. It appears that in the main the total radiation is a function of the mass. Stars of equal mass but different density, and consequently different spectral type or effective temperature, will show minor differences of brightness which will be ignored in the present brief account of the results; they have, however, been duly allowed for in the comparisons of theory and observation referred to below and illustrated in the diagram. With this understanding we shall regard the absolute brightness as a function of the mass only-always provided that the star is not too dense to be treated as a perfect gas.

The theory divides itself into two parts, (I) the determination of the internal temperature-distribution leading to a knowledge of the temperature-gradient which causes the outward flow of radiation through the star, and (2) the determination of the coefficient of absorption or opacity obstructing this outward flow. The first part now presents no serious difficulty; the solution follows the classical researches of Lane, Ritter, Emden, and others, modified by introducing radiative instead of convective equilibrium and taking account of radiation-pressure. It is necessary to know the average molecular weight of the material ; owing to ionisation this is smaller than the atomic weight, because each atom is split up into a number of independently moving particles (nucleus and electrons). By actual calculation we find that in all ordinary stars the molecular weight is nearly $2-$ a result practically independent of the chemical composition of the material. The second part of the theory leads us to one of the most fundamental problems of modern atomic physics, the absorption of X-rays by matter, and the capture of electrons by ionised atoms-in fact, the general problem of interaction of radiation and material systems. Although the theory of these processes is as yet scarcely definitive, very general considerations lead to the result that the coefficient of absorption $k$ will be proportional to $\rho / \mathrm{T}^{2}$, subject perhaps to a small correcting term which may be ignored in a first approximation. The important theory recently published by H. A. Kramers (Phil. Mag., November I923) agrees with this law.

The result of the theoretical discussion is that we obtain for the absolute magnitude $m$ of the star a formula of the form

$$
m=f(\mathrm{M})+\text { constant }
$$

where $f(\mathrm{M})$ is a definite function of the mass $\mathrm{M}$ which can be tabulated, and all the doubtful constants and theoretical uncertainties are segregated in the value of the additive constant. The predicted value of this constant is certainly of the right order of magnitude as compared with astronomical observation, but we cannot at present see the way clear to reach satis- factorily the exact value. We shall shelve this diffculty by determining the value of the constant from the astronomical observations of Capella, for which accurate values of $m$ and $\mathrm{M}$ are known. The results for all other stars will then depend differentially on Capella. The theoretical curve connecting absolute magnitude and logarithm of mass is shown in the diagram (Fig. r). All the observed data that could be collected are inserted for comparison with it. The circles and crosses correspond to direct determinations, the circles being the more accurate. The determinations from eclipsing variables, represented by triangles, are less direct but involve only obvious theoretical inferences; owing to practical difficulties they are not in all cases quite satisfactory. The squares depend on a definite theory of the mechanism of Cepheid variation which would perhaps not commend itself to every one, and the value of their evidence may be a matter of controversy.

The curve shown is a first approximation and involves only two disposable constants, (I) the additive constant deduced from Capella, and (2) the molecular weight here taken to be $\mathbf{2}^{\prime} \mathrm{I}$ for the reasons already stated. Certain refinements leading to a second approximation would leave the greater part of the curve unaltered, but would raise it about $\mathrm{I} \frac{1}{2}$ magnitudes at the extreme left of the diagram, giving a slightly better accordance with observation. Taking the first approximation as it stands, the average discordance is $\pm 0^{m \cdot} 5^{6}$, the greater part of which may well be attributable to observational errors. The only stars rejected are two "white dwarfs" which are clearly in a state quite outside the conditions postulated in the theory.

The remarkable thing is that although the theoretical curve corresponds to stars in the condition of (i.e. having the compressibility of) a perfect gas, all ordinary stars agree with it very closely. All the stars in the left half of the diagram are dwarf stars with a mean density comparable with water. Consider, for example, the sun-represented by the circle on the vertical line through 0.0 in the diagram. According to the current giant and dwarf theory, a star of mass I and effective temperature $5800^{\circ}$ has two possible luminosities, (I) that of the sun in its present condition, (2) that of the sun at an earlier epoch when it passed through the same temperature on the upgrade as a much more diffuse star. It has generally been supposed that there would be about 4 magnitudes difference between these two stages corresponding to the change of surface-area. The theory is intended to predict No. 2 , but actually predicts No. $x$.

In making the comparisons for these dense stars the writer had no anticipation that they would agree with the curve ; his object was rather to obtain exact data as to the diminution of brightness attributable to the reduced compressibility to be expected in gas at high density, and he felt the temporary disappointment which naturally arises from a negative result. If we are to accept these results at their face value it would appear that these stars have the luminosity predicted for a perfect gas because their material actually has

$$
\text { NO. } 2848 \text {, VOL. II } 3 \text { ] }
$$


the compressibility of a perfect gas-notwithstanding that some of them have mean densities as great as that of platinum.

Such a conclusion may perhaps appear incredible, and point to some false step in the rather intricate theoretical investigation. But I think, on the other hand, that we have only been led in a very roundabout way to a conclusion which is almost obvious from modern physical theory, though it does not seem to have occurred to any one. An ordinary gas becomes comparatively incompressible at high density because of the finite volume occupied by its atoms or molecules ; these behave like rigid spheres with radii of the order $10^{-8} \mathrm{~cm}$., and a limit to the possible compression is reached when the spheres become tightly packed. terrestrial conditions; the ions, or broken atoms, can be packed much more tightly. For a complete discussion it is necessary to calculate the effect of the strong electrostatic forces acting between the charged ions and electrons in the star; it appears, however, that these forces will not have an appreciable effect in any of the stars under consideration.

Our results, therefore, may well be acceptable to physicists; they are less welcome to astronomers on account of their conflict with the giant and dwarf theory of evolution. As already mentioned, the latter theory requires that absolute magnitude should be a double-valued function of mass; the observational evidence summarised in the diagram (considered without regard to the theoretical curve) strongly

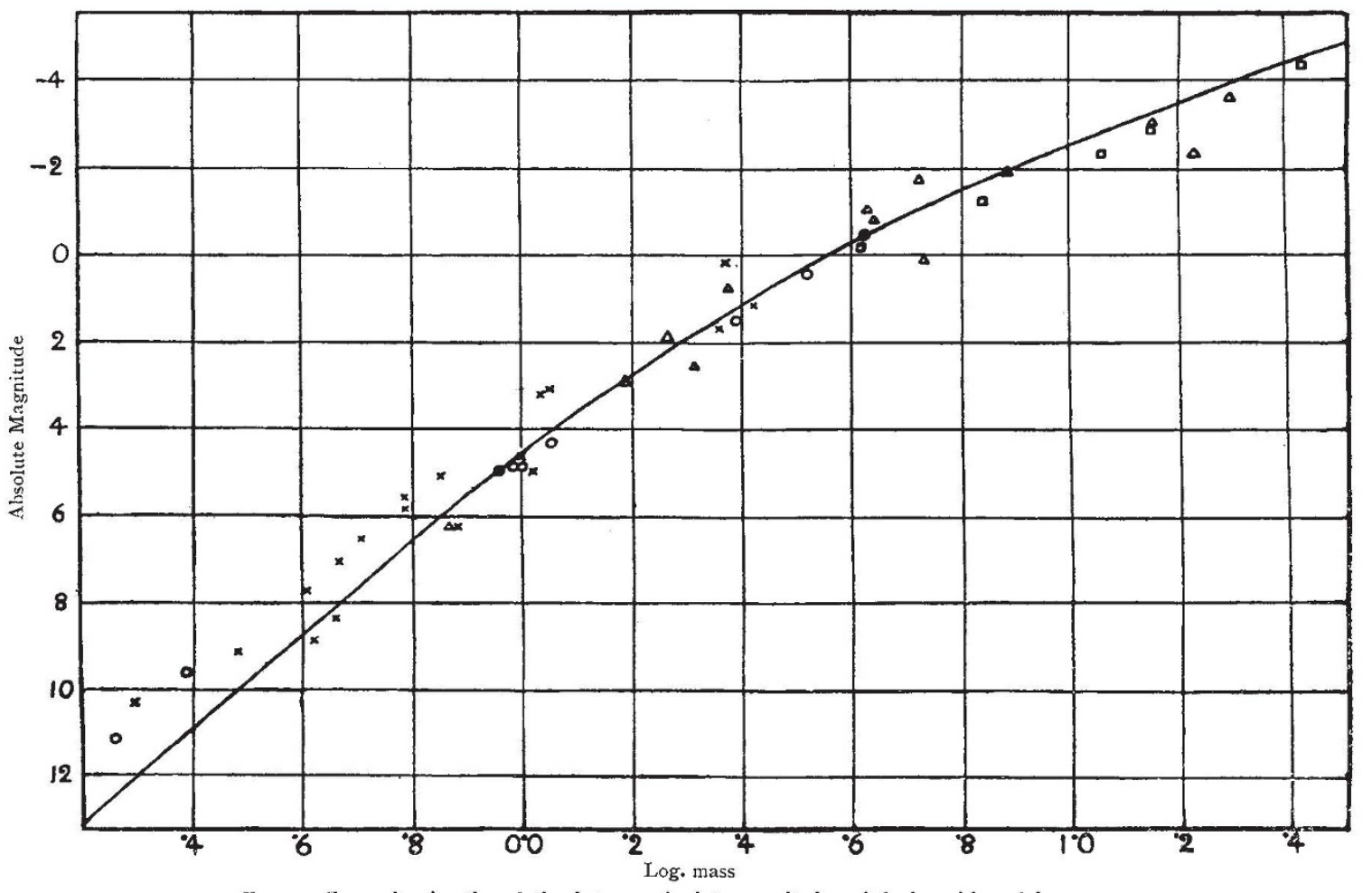

Fig. I. - Curve showing the relation between absolute magnitude and the logarithm of the mass.

0 , first class; $x$, second class; $\square$, Cepheids; $\Delta$, eclipsing variables.

But at the high temperature within a star these spheres are completely destroyed, and this limit to the compression disappears. The stellar atom is highly ionised, and the peripheral electrons which determine its effective size have been detached. For elements of moderate atomic weight (e.g. iron) only the two $\mathrm{K}$ electrons remain, and the lighter elements (e.g. oxygen) are ionised down to the bare nucleus. If the effective size of the atom is still determined by the orbits of the outermost electrons remaining, the stellar atom cannot have more than $\mathrm{r} / \mathrm{roo}, 00 \mathrm{oth}$ of the bulk of the atom in terrestrial conditions. Consequently, we should expect deviations from the laws of a perfect gas to begin at densities about 100,000 times higher. At any rate our preconceived idea that stellar material of the density of platinum cannot be compressed like a perfect gas, seems to rest on an entirely false analogy between stellar ions and the atoms met with in suggests a single-valued function, as has also been indicated by recent investigations of Hertzsprung and of Russell, Adams, and Joy. Although data as to masses of stars are scanty, it is unlikely that a second branch would have been unrepresented if it existed. There is thus a strong case for a fundamental modification of the giant and dwarf theory of evolution. On the other hand, important advances in our general knowledge of the stars are associated with the giant and dwarf theory, and it has been fertile in conclusions clearly confirmed by observation. The astronomer will no doubt wish to convince himself that these undoubted advances can be retained in the reconstructed theory of evolution before committing himself to acceptance of the result here reached.

If stellar material can still be a perfect gas when compressed to the density of platinum, the maximum density must be a great deal higher. There might

NO. 2848 , vOL. I I 3$]$ 
thus exist stars far more dense than any material yet known to us. This may be the key to a puzzle presented by the companion of Sirius and a few other stars known as "white dwarfs"; the newly discovered companion of Mira Ceti is (according to preliminary accounts) another example. The faint companion of Sirius is classed as of spectral type $F$; if this means that it has the surface-brightness ordinarily associated with type $\mathrm{F}$, its radius is about 20,000 kilometreswhich seems absurdly small for a star. Its mass is well-determined and is about $\frac{4}{5}$ that of the sun. The resulting mean density is $50,000 \mathrm{gm}$. per c.c. This conclusion has probably been regarded hitherto as a reductio ad absurdum, showing that the star must manage in a mysterious way to produce the $\mathrm{F}$ type of spectrum without the intensity of radiation ordinarily required for it. But the deduced density cannot now be considered impossible, and the companion of Sirius may be an actual example of the high density attainable by matter when ionised by the enormous temperature in a star. Fortunately it will be possible to test whether this is so by determining the Einstein shift of the spectral lines, which would be large enough to be measured if the high density is correct. If the result of the test should prove favourable and establish the existence of matter having density of the order 50,000 , we shall have fewer qualms in accepting perfect gases of the density of platinum.

\section{Electrical Exhibits at the British Empire Exhibition.}

THE greater part of the exhibits of electrical interest in the British Empire Exhibition at Wembley Park is concentrated in the great Palace of Engineering, which, during these early weeks of the Exhibition, is one of the most complete portions-and perhaps the most imposing-of the whole stupendous display. The electrical engineering section, which was organised by the British Electrical and Allied Manufacturers' Association, in conjunction, as regards certain sections, with the Electrical Development Association and the Cable Makers' Association, covers no less than $4 \frac{1}{2}$ acres of the vast building, forming, we believe, the largest single section of the Exhibition and probably the most complete collection of modern electrical engineering plant and apparatus that has yet been brought together. It is concentrated in one end of the building, and provided with entrances named appropriately after Faraday and Kelvin.

An important feature of the heavy electrical plant is the complete generating station, used to supply power to the Exhibition, with fully equipped boiler-house, turbo-generators, switchgear, transformers, etc., representing some 6000 h.p., all in full view of visitors. Generating plant, on a large scale driven by turbines, reciprocating steam engines, and internal combustion engines, is also represented on the stands of practically all the well-known British makers of such plant. We do not propose to refer here to more than two or three examples of the exhibits of those firms the names of which are household words to every electrical engineer, including, besides those mentioned below, such firms as the English Electric Co., Ltd., the MetropolitanVickers Electrical Co., Ltd., the General Electric Co., Ltd., the Brush Electrical Engineering Co., Ltd., Crompton and Co., Ltd., Bruce Peebles and Co., Ltd., Mather and Platt, Ltd., the British Electric Transformer Co., Ltd., Electromotors, Ltd., and many others.

From the steam side, one of the most interesting of these exhibits is the $12,000 \mathrm{kw}$. turbo-generator of the latest type of C. A. Parsons and Co., Ltd., marking the culmination of many years of development in generating plant. From the point of view of hydroelectric power development, an outstanding exhibit is a 30,000 h.p. twin impulse turbine to run at 375 r.p.m. under a head of $1630 \mathrm{ft}$., with the Seewar patent governing device shown in action by means of a separate, electrically driven oil-pump, forming part of the comprehensive display of the English Electric Co., Ltd. Five such turbines are now in course of manufacture for India. An interesting exhibit on the purely electrical side is the display of a complete, automatically controlled, $500 \mathrm{kw}$. rotary converter substation on the large stand of the British Thomson-Houston Co., Ltd. This exemplifies a modern tendency much on the increase, particularly in high-tension D.C. railway working. Another sign of recent developments, relating, however, more to overseas conditions than to those at home, is the presence of apparatus for outdoor substations and switch-points of very high tension transmission lines. Here it is interesting to note that Electric Control, Ltd., has provided an alternative to the usual oil-break apparatus in air-break switches for voltages up to I 30,000 volts. This firm also shows air-break fuses for overhead open-air positions for such high voltages, and an interesting fuse, enclosed in a glass tube filled with carbon tetrachloride, which immediately extinguishes the arc without the glass tube breaking. Modern developments in another direction, that of power-factor compensation, are exemplified in the display by British Insulated and Helsby Cables, Ltd., of electrostatic condensers for this purpose, capable of dealing with as much as $300 \mathrm{kva}$. in one unit.

The two principal large exhibits relating to electric traction are a train exhibited by the Metropolitan Railway in the Palace of Engineering and the electric locomotive attached to the South African train in another part of the grounds. Electric working in collieries, including a complete winding plant, will be found in operation in the model coal mine. These features we have not space to describe here.

Switchgear and motor control gear are exhibited by firms too numerous to particularise. Perhaps the finest display of this kind is that of iron-clad switchgear by Reyrolle and Co. Turning from large to small apparatus, a very convenient type of commutating rectifier may be mentioned on the stand of the Lancashire Dynamo and Motor Co., Ltd., associated with the Crypto Electrical Co., Ltd. This is for charging small accumulators, running cinema arc lamps, or other D.C. apparatus from A.C. circuits, and comprises, all in one compact little machine, a transformer and a synchronous motor, with an external commutator provided with suitable brush gear and arranged with a self-starting

No. 2848 , voL. I I 3$]$ 